\title{
Papel del túbulo en la vasoconstricción renal inducida por ciclosporina
}

\author{
Camaño Páez S*, Lázaro Fernández A*, Callejas Martínez R*, Lázaro Manero JA*, \\ Castilla Barba $\mathrm{M}^{*}$, Martín-Vasallo $\mathrm{P}^{* *}$, Martínez Escandell $\mathrm{A}^{* * *}$, Tejedor Jorge $\mathrm{A}^{*}$. \\ *Servicio de Nefrología. Laboratorio de Fisiopatología Renal. Hospital General Univ. Gregorio Marañón. Madrid. \\ **Departamento de Bioquimica y Biología Molecular. Universidad de la Laguna. Tenerife. \\ ***Disease of the Developing World Center Laboratory. Animal Science Department. \\ Glaxo Smith Kline I+D. España.
}

(En representación del Consorcio del Fracaso Renal Agudo. Comunidad de Madrid).

Actas Urol Esp. 2008;32(1):128-139

\section{RESUMEN}

PAPEL DEL TÚBULO EN LA VASOCONSTRICCIÓN RENAL INDUCIDA POR CICLOSPORINA

Introducción: Ciclosporina es el primero de una familia de potentes inmunosupresores con capacidad anticalcineurinica que, sin embargo, presentan como limitación terapéutica una nefrotoxicidad que puede aparecer desde periodos tempranos. Los primeros datos funcionales sugerian la existencia de vasoconstricción renal, si bien estudios posteriores han mostrado un efecto tóxico directo de ciclosporina sobre el túbulo proximal.

Materiales y Métodos: En este artículo se presenta un modelo porcino de nefrotoxicidad por ciclosporina a medio plazo, analizándose los cambios hemodinámicos intrarrenales y las funciones tubulares, así como la relación entre ambos.

Resultados: Ciclosporina produce específicamente una internalización e inactivación de la $\mathrm{Na}^{+}, \mathrm{K}^{+}$-ATPasa basolateral del túbulo proximal, determinando el desplazamiento axial de la carga filtrada hacia regiones distales (asa de Henle), con transportes activos más intensos y no modificados por ciclosporina. El resultado es un aumento en la reabsorción fraccional distal de cloro que condiciona un estímulo del "feed-back" túbulo glomerular, vasoconstricción aferente pero no eferente, aumento de las resistencias renales y caída de la presión intraglomerular. Furosemida restaura parcialmente la situación basal. La consecuencia inmediata es un aumento en el consumo de $\mathrm{O}_{2}$ por unidad de sodio trasportado. A nivel del túbulo colector no se observan cambios en la secreción de agua libre, pero la secreción de $\mathrm{K}^{+}$ parece limitada, y no se corrige al aumentar la oferta distal de sodio con furosemida, ni con aldosterona, cuyos valores fueron más altos en los animales tratados con ciclosporina.

Conclusión: En resumen, el efecto vasoconstrictor de ciclosporina sobre el riñón se debe en parte a una retroalimentación túbulo glomerular activada por el desplazamiento de solutos filtrados de sus sitios de reabsorción proximal a otros sitos más distales. Las dietas con alto contenido en sodio pueden aumentar el efecto deletéreo de ciclosporina sobre el filtrado glomerular.

Palabras clave: Ciclosporina. Nefrotoxicidad. Furosemida. $\mathrm{Na}^{+}, \mathrm{K}^{+}$-ATPasa.

\section{ABSTRACT}

STUDY ON THE ROLE OF THE TUBULE IN RENAL VASOCONSTRICTION INDUCED BY CYCLOSPORINE

Introduction: Cyclosporine (CyA) has proved to induce cell apoptosis on cultured proximal tubule cells. However, there is no much data about the in vivo functional consequences of this injury or the long time observed CyA-induced renal vasoconstriction.

Material and Methods: In a swine model of subacute CyA nephrotoxicity (10 mg/ Kg. d x 15 days), we performed a right nephrectomy, followed by left renal artery, vein and ureter catheterisation. After inducing water diuresis, three clearance periods of 15 minutes were performed before and after a furosemide $1 \mathrm{mg} / \mathrm{kg}$ infusion. Plasma and urine electrolytes, blood gas, acid excretion, plasma renin activity and aldosterone concentration, GFR, RPF, RBF, intra-renal vascular resistances, glomerular filtration pressure, distal $\mathrm{Cl}^{-}$delivery, water clearance and TTKG were measured or estimated on 7 control and 7 treated animals. Right kidney was processed for NaKATPase activity and immunostaining.

Results: Treated animals presented detaching proximal cells, luminal blebbing and loss of tight junctions. Cortical but not medullar sodium pump was internalised and partially inactive. Treated animals showed much lower fractional excretions of $\mathrm{Na}^{+}$, with significantly higher distal fractional reabsorption of $\mathrm{Cl}^{-}$. Distal shift in fluid load resulted in a significant rise in renal $\mathrm{O}_{2}$ consumption, and modifications in the global renal estequiometry of $\mathrm{Na}^{+}$transport $/ \mathrm{O}_{2}$ uptake. Several consequences followed this situation: preglomerular resistances increased 3 times with only minor changes in postglomerular resistances and renal blood and plasma flow were significantly reduced. Furosemide partially reversed these effects. A slight increase in fractional filtration prevented GFR differences to become statistically significant.

Conclusión: subacute CyA treatment even al doses not modifying GFR, may cause proximal tubule $\mathrm{Na}^{+}$transport impairment, resulting in increased rates of distal delivery and absorption of fluid load. Renal uptake of $\mathrm{O}_{2}$ may be increased and tubule glomerular feedback should be expected to be activated. Absence of changes of GFR with furosemide is an early sign of CyA renal damage.

Keywords: Cyclosporine. Nephrotoxicity. Furosemide. $\mathrm{Na}^{+}, \mathrm{K}^{+}$-ATPase. 
$\mathrm{D}$ esde su introducción en clínica, los inmunosupresores anticalcineurínicos como la ciclosporina (CsA) han revolucionado el tratamiento de los trasplantes de órganos sólidos y algunas enfermedades autoinmunes, al suprimir de modo reversible la sintesis y secreción de interleukina 2 por los linfocitos $\mathrm{T}^{1}$.

Sin embargo su uso clínico se ha visto ensombrecido por la aparición de varios efectos secundarios, de los que el más grave ha sido sin duda la nefrotoxicidad. Aunque en la actualidad se acepta ampliamente que el principal daño primario es sobre el túbulo proximal, donde CsA causa apoptosis $^{2}$, las primeras descripciones de la nefrotoxicidad por CsA mostraron un aumento de las resistencias intrarrenales. Aún hoy en día es una opinión habitual atribuir a la isquemia renal las lesiones tubulares observadas. Aunque la demostración de que CsA es tóxica incluso sobre cultivos primarios de células tubulares, en ausencia de isquemia o hipoxia, y que dicha lesión reduce la actividad de la NOS 1 y NOS 2 de la célula proximal ${ }^{3}$, nunca se ha podido establecer un nexo de unión entre la lesión tubular y los cambios hemodinámicos descritos. Por otro lado, tampoco existe una descripción clara de dichos cambios hemodinámicos.

El objetivo de este trabajo ha sido reproducir un modelo animal (porcino) de toxicidad subaguda por CsA, para caracterizar los principales aspectos funcionales hemodinámicos y tubulares del mismo, buscando establecer las bases fisiopatológicas del fracaso renal en dicho contexto.

\section{MATERIALES Y MÉTODOS}

\section{Modelo animal}

El modo de toxicidad subaguda por CsA se llevó a cabo sobre 14 cerdos miniatura de estirpe Maryland, con una edad de 3 meses y un peso medio de $29,3 \pm 1,4 \mathrm{Kg}$, isogénicos para el loci del complejo mayor de histocompatibilidad ${ }^{4}$. Todos los estudios se han hecho de acuerdo con las normas éticas de la Comunidad Europea (Directiva del Consejo de Europa 86/609/ CEE-Leg CCEE 4390), y las direc- trices del Real Decreto 223/4988 (R601), y fueron aprobadas por el Comité de Investigación del Hospital.

La mitad de los animales recibieron CsA (Sandimun ${ }^{\circledR}$ oral) durante 15 días. La CsA se añadió a la comida en una sola dosis de $10 \mathrm{mg} /$ $\mathrm{Kg} /$ día, asegurándose de que los animales consumian la totalidad de la comida tratada. Las condiciones de nutrición, acceso al agua y estabulación fueron igual para los animales control y los sometidos a tratamiento con CsA.

Al cabo de 15 días los animales fueron premedicados con Ketolar $10 \mathrm{mg} / \mathrm{Kg}$, Rompun $1 \mathrm{mg} / \mathrm{Kg}$ y atropina $0,025 \mathrm{mg} / \mathrm{Kg} \mathrm{im}$. La anestesia se indujo con pentotal sódico $20 \mathrm{mg} / \mathrm{Kg}$ iv y se mantuvo con $\mathrm{N}_{2} \mathrm{O}: \mathrm{O}_{2}$ (4:2), Fentanest y Pavulón.

Durante la cirugia se procedió a la nefrectomía derecha y colocación de catéteres en aorta, vena renal izquierda y uréter izquierdo para la obtención de medidas hemodinámicas y muestras de sangre. Tras un periodo de 10 min de estabilización se iniciaba una perfusión de suero hiposalino a $4 \mathrm{~mL} /$ min destinada a obtener una diuresis venosa. El diseño experimental (Fig. 1) constó de 3 periodos de aclaramiento de 15 min de duración, seguidos de la administración de furosemida $1 \mathrm{mg} / \mathrm{Kg}$ en bolo, y 3 periodos más de aclaramiento de 15 min cada uno. Al inicio y final de cada periodo se obtuvieron muestras de sangre de la aorta y la vena renal, y se cuantificó la orina emitida. Se obtuvieron asimismo las presiones en la aorta y vena renal.

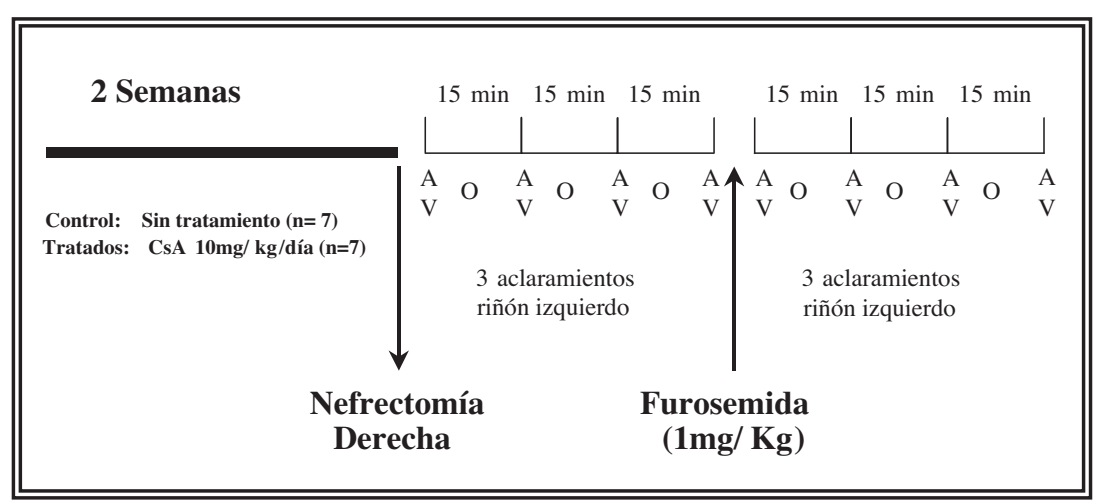

FIGURA 1. Diagrama del diseño experimental. 7 animales control y 7 animales tratados con CsA $10 \mathrm{mg} / \mathrm{Kg}$.dia por via oral, fueron estabulados durante 15 dias antes del estudio. El estudio consistió en 3 periodos de aclaramiento de 15 minutos cada uno tras la realización de nefrectomia unilateral, seguido de la infusión de furosemida $1 \mathrm{mg} / \mathrm{Kg}$ y otros tres periodos de aclaramiento idénticos. Al inicio y al final de cada periodo de aclaramiento se obtuvieron muestras de sangre de la aorta (A) y de la vena renal (V). Asimismo se recolectó toda la orina (O) durante el mismo periodo (ver Materiales y Métodos). 


\section{Procesamiento de muestras de sangre y orina}

Se obtuvieron $15 \mathrm{~mL}$ de sangre arterial y venosa, realizándose determinaciones de gasometria, hematocrito y hemoglobina en sangre completa, y creatinina, sodio, potasio, cloro, CsA y osmolaridad en plasma tras separación del mismo por centrifugación a $3000 \mathrm{rpm}$ durante $10 \mathrm{~min}$.

La orina se recogió bajo $3 \mathrm{~mL}$ de aceite de parafina para evitar pérdidas por evaporación. Sobre las muestras se determinó volumetría, gasometría, acidez titulable, $\mathrm{pH}$ y concentración de sodio, cloro, creatinina y osmolaridad.

La actividad de renina plasmática y aldosterona se midieron por radioinmunoensayo (RIA), y CsA por TDX (Abbot Laboratoires, USA) ${ }^{2}$. El resto de parámetros se determinó de modo automatizado en un Autoanalizador Analítico Modular SWA (Hitachi-Roche) y un Hemocitómetro Analizador LH750 (Beckman-Coulter).

\section{Parámetros estimados}

El filtrado glomerular (FG) se estimó a partir del aclaramiento de creatinina $(\mathrm{ClCr})$. El flujo plasmático renal (FPR), a partir del FG y la excreción renal de creatina:

$$
\mathrm{FPR}=\frac{\mathrm{ClCr}}{(\mathrm{CrA}-\mathrm{CrV}) / \mathrm{CrA}}
$$

El flujo sanguíneo renal (FSR) se estimó a partir del FPR y el hematocrito. La fracción de filtración se calculó como el FG/FPR, expresado en porcentaje.

El estudio de la hemodinámica intraglomerular se basó en las ecuaciones de Gómez ${ }^{5}$, ampliamente distribuidas y validadas por otros ${ }^{6}$. La resistencia renal total se obtuvo de la determinación directa de la presión arterial (PA) en la aorta y la estimación del FSR. Las resistencias en la arteriola aferente se estimaron a partir de la presión en el glomérulo, según el método indicado $^{5}$. Las resistencias en la arteriola eferente se obtuvieron a partir de los valores de presión glomerular y de presión en la vena renal, asumiendo que la resistencia en las venas pequeñas intrarrenales es prácticamente cero, como ha sido demostrado ${ }^{5}$.
La excreción fraccional (EF) de los iones se estimó como la fracción de la carga filtrada que es eliminada por orina, expresada en forma de porcentaje:

$$
\text { Efion }=\frac{[\text { ion }]_{\mathrm{o}} \times \text { Vdiuresis }}{[\text { ion }]_{\mathrm{p}} \times \mathrm{FG}}=\frac{[\text { ion }]_{\mathrm{o}} \times[\mathrm{crea}]_{\mathrm{p}}}{[\text { ion }]_{\mathrm{p}} \times[\mathrm{crea}]_{\mathrm{o}}}
$$

La excreción fraccional de agua es el volumen de diuresis expresado como porcentaje del FG.

El aclaramiento de agua libre es el agua que hay que añadir o sustraer al aclaramiento osmolar para obtener un volumen equivalente a la diuresis, y se obtiene sustrayendo del volumen de diuresis el aclaramiento osmolar.

La reabsorción fraccional de cloro de la nefrona distal ( $\mathrm{RFdCl})$ se estimó como la fracción del cloro reabsorbido que da lugar a la formación de agua libre, ya que dicho proceso sólo tiene lugar a nivel de la nefrona distal. Se expresa en porcentaje:

$$
\mathrm{RFdCl}=\frac{\mathrm{Cl} \mathrm{H} 2 \mathrm{O} \text { libre }}{(\mathrm{Cl} \text { H2O libre }+\mathrm{Cl} \text { Cloro })} \times 100
$$

El gradiente transtubular de $\mathrm{K}^{+}$(GTTK) representa el número de veces que la concentración del Kp aumenta a nivel del túbulo colector cortical, y depende de la oferta de $\mathrm{Na}^{+}$urinario a dicho nivel, y de la actividad de la aldosterona. Su cálculo necesita estimar la concentración de $\mathrm{K}^{+}$en la luz del túbulo, a partir del $\mathrm{K}^{+}$urinario, $\mathrm{y}$ la osmolaridad en orina y plasma ${ }^{7}$ :

$$
\text { GTTK }=\frac{[\mathrm{K}]_{\mathrm{o}} \times \operatorname{osm}_{\mathrm{p}} / \operatorname{osm}_{\mathrm{o}}}{[\mathrm{k}]_{\text {plasma }}}=\frac{[\mathrm{K}]_{\text {orina }} \times \operatorname{osm}_{\mathrm{p}}}{[\mathrm{K}]_{\text {plasma }} \times \text { osm }_{\mathrm{o}}}
$$

Un valor superior a 7 indica una hipersecreción de $\mathrm{K}^{+}$(hiperaldosteronismo), mientras que inferior a 4 indica una reducción del gradiente por hiposecreción de $\mathrm{K}^{+}$(hipoaldosteronismo) o por aumento del flujo urinario a dicho nivel.

El contenido total de oxígeno se calculó teniendo en cuenta las saturaciones de $\mathrm{O}_{2}$ de la hemoglobina en la aorta y la vena renal y la concentración de hemoglobina en ambas. Asumiendo que $1 \mathrm{~g}$ de hemoglobina saturada al 100\% contiene $1,34 \mathrm{~mL}$ de $\mathrm{O}_{2}$, y que $22,4 \mathrm{~L} / \mathrm{mol}$ es el volumen molar de los gases: 
$\left(\mathrm{Hgb} \times\right.$ Sat $\left.\mathrm{O}_{2}\right) \times 1,34$ mmoles/mLsangre Contenido en $\mathrm{O}_{2}=\frac{224}{224}$

A partir del contenido en $\mathrm{O}_{2}$ en la arteria (aorta) y vena renal se obtiene el consumo de oxígeno:

(contenido $\mathrm{O}_{2}$ arteria) - (contenido $\mathrm{O}_{2}$ vena)

Consumo real de $\mathrm{O}_{2}=\frac{(\text { FSR }}{\text { FStenido }}$

\section{Actividad de la $\mathrm{Na}^{+}, \mathrm{K}^{+}$-ATPasa}

$\mathrm{La}$ actividad máxima $\mathrm{Na}^{+}, \mathrm{K}^{+}$-ATPasa se determinó sobre microsomas corticales y medulares renales de animales control y tratados durante 15 días con CsA 10 mg/Kg.día, y se obtuvo como actividad ATPásica sensible a ouabaína ${ }^{8}$. El medio de ensayo contiene imidazol $25 \mathrm{mM}$, histidina $25 \mathrm{mM}$, EDTA $1 \mathrm{mM}, \mathrm{NaCl} 100 \mathrm{mM}, \mathrm{KCl} 5 \mathrm{mM}, \mathrm{MgCl}_{2}$ $1 \mathrm{mM}, \pm$ ouabaína $1 \mathrm{mM}$. El sistema auxiliar contiene fosfoenol piruvato $1 \mathrm{mM}$, NADH 0,35 mM, LDH y piruvato kinasa, acoplándose la aparición específica de ADP a la transformación de fosfoenol piruvato en piruvato. La formación de ADP se cuantificó mediante la reducción de piruvato a lactato por $\mathrm{LDH}$, en presencia de $\mathrm{NADH}^{8}$.

\section{Distribución e inmunolocalización celular de las subunidades $\alpha 1, \beta 1, y \gamma$ de la $\mathrm{Na}^{+}, \mathrm{K}^{+}$- ATPasa renal}

Las inmunolocalizaciones fueron realizadas en secciones renales de $4 \mu \mathrm{m}$ de grosor incluidas en parafina procedentes de los riñones derechos de los animales control y tratados con CsA, que fueron previamente desparafinadas y rehidratadas en concentraciones decrecientes de etanol. Posteriormente se permeabilizó el tejido con PBS-0,2\% de Triton X-100, y se bloquearon en PBS-1\% BSA durante 10 min cada uno. Después de lavar el tejido con PBS, los cortes fueron incubados toda la noche a a $4^{\circ} \mathrm{C}$ con los anticuerpos primarios Mck 1 (para $\alpha 1$; dilución $1: 10$ ), SpETb1 (para $\beta 1$; dilución 1:500) y G17 (para $\gamma$; dilución 1:20) en PBS-1\% BSA. Los anticuerpos fueron cedidos amablemente por los Drs. KJ Sweadner, P. Martín Vasallo y RW Mercer respectivamente ${ }^{9-11}$. Tras lavar varias veces con PBS, se incubó durante $1 \mathrm{~h}$ con el anticuerpo secundario marcado apropiadamente. Los núcleos celulares fueron teñidos con DAPI.

\section{Método estadístico}

De los 7 animales asignados al grupo control y al grupo tratado con CsA, uno del último grupo no pudo ser incluido por problemas técnicos durante la cirugía. Los demás completaron el protocolo y se incluyeron en el estudio.

Los datos se presentan como la media \pm EEM. La comparación de variables entre los grupos control y CsA se realizó mediante una $t$ de Student para datos apareados, pero cuando es estudiado el efecto del tratamiento sobre la respuesta a furosemida, se aplicó un ANOVA para medidas repetidas con 2 factores (furosemida y CsA), estudiando el modelo con interacción entre ambos factores.

En los estudios de regresión se han utilizado técnicas convencionales de regresión y comparación de coeficientes mediante la $t$ de Student.

Se han considerado significativas diferencias con un $\alpha$ bilateral inferior a 0,05.

\section{RESULTADOS}

\section{Parámetros bioquímicos}

A los 15 dias de tratamiento, los animales tratados con CsA presentaban una concentración de la misma en sangre de $1370 \mathrm{ng} / \mathrm{mL}$ (Tabla 1). No se observaron diferencias en las concentraciones plasmáticas de $\mathrm{Na}^{+}, \mathrm{K}^{+}, \mathrm{Cl}^{-}$, bicarbonato o en la osmolaridad. La creatinina plasmática fue significativamente mayor en los animales tratados con CsA respecto a los controles.

La actividad de renina plasmática y la concentración de aldosterona en plasma fueron significativamente más altas en el grupo tratado con CsA (Tabla 1).

Las excreciones urinarias de $\mathrm{Na}^{+} \mathrm{y} \mathrm{Cl}^{-}$fueron significativamente más bajas, casi 10 veces inferiores a los del grupo control. No se observaron diferencias en la excreción neta de ácido ni en sus componentes (Tabla 1).

\section{Parámetros hemodinámicos}

La PA media medida a nivel aórtico en los animales control fue de $139,2 \pm 7,2 \mathrm{~mm} \mathrm{Hg}$ frente a los animales tratados con CsA: 132,1 $\pm 5,45 \mathrm{~mm}$ $\mathrm{Hg}$ (NS).

El FSR y el FPR se redujeron significativamente en los animales tratados con CsA respecto a los animales control (Figs. 2A y 2B). 
Tabla 1. Parámetros bioquímicos en plasma y orina.

\begin{tabular}{|c|c|c|c|}
\hline $\begin{array}{l}\text { Parámetros en } \\
\text { Plasma }\end{array}$ & Control & Ciclosporina & $\mathbf{P}$ \\
\hline $\mathrm{CsA}(\mathrm{ng} / \mathrm{mL})$ & - & $1370 \pm 242,3$ & - \\
\hline $\mathrm{Na}(\mathrm{mEq} / \mathrm{L})$ & $145,5 \pm 2,1$ & $145,6 \pm 3,9$ & - \\
\hline $\mathrm{K}(\mathrm{mEq} / \mathrm{L})$ & $3,4 \pm 0,2$ & $3,8 \pm 0,1$ & - \\
\hline $\mathrm{Cl}(\mathrm{mEq} / \mathrm{L})$ & $113,9 \pm 1,1$ & $112 \pm 1,7$ & - \\
\hline $\mathrm{HCO}_{3}(\mathrm{mEq} / \mathrm{L})$ & $25,2 \pm 1,0$ & $22,8 \pm 1,0$ & - \\
\hline Osm (mosmol/L) & $305,2 \pm 10,5$ & $301,8 \pm 2,7$ & - \\
\hline Creatinina $(\mathrm{A})(\mathrm{mg} / \mathrm{dL})$ & $1,0 \pm 0,05$ & $1,3 \pm 0,1$ & $<0,05$ \\
\hline Creatinina $(\mathrm{V})(\mathrm{mg} / \mathrm{dL})$ & $0,8 \pm 0,05$ & $0,9 \pm 0,1$ & $<0,1$ \\
\hline $\begin{array}{l}\text { Actividad de Renina } \\
\text { (ng/mL.h) }\end{array}$ & $0,4 \pm 0,1$ & $2,3 \pm 1,1$ & $<0,05$ \\
\hline Aldosterona (pg/mL) & $<50$ & $93,5 \pm 30,1$ & $<0,05$ \\
\hline Hemoglobina $(\mathrm{g} / \mathrm{dL})$ & $10,4 \pm 0,5$ & $14,4 \pm 1,1$ & $<0,05$ \\
\hline $\begin{array}{l}\text { Presión Arterial Media } \\
(\mathrm{mm} \mathrm{Hg})\end{array}$ & $139,2 \pm 7,2$ & $132,1 \pm 5,5$ & - \\
\hline $\begin{array}{l}\text { Parámetros en } \\
\text { Orina }\end{array}$ & Control & Ciclosporina & $\mathbf{P}$ \\
\hline $\mathrm{Na}(\mathrm{mEq} / \mathrm{min})$ & $95,8 \pm 61,2$ & $8,7 \pm 3,6$ & $<0,05$ \\
\hline $\mathrm{K}(\mathrm{mEq} / \mathrm{min})$ & $19,3 \pm 3,7$ & $11,5 \pm 4,1$ & - \\
\hline $\mathrm{Cl}$ (mEq/min) & $117,6 \pm 68,2$ & $17,3 \pm 5,5$ & $<0,05$ \\
\hline $\mathrm{HCO}_{3}(\mathrm{mEq} / \mathrm{min})$ & $1,3 \pm 3,2$ & $0,6 \pm 0,5$ & - \\
\hline $\mathrm{HH}_{4}(\mathrm{mEq} / \mathrm{min})$ & $7,6 \pm 3,2$ & $6,7 \pm 1,2$ & - \\
\hline
\end{tabular}

como retroalimentación o "feed-back" túbulo glomerular. En él, cuando hay un aumento en el trasporte de sodio a través de las células de la mácula densa, dichas células segregan mediadores del tipo de la adenosina que provocan la vasoconstricción de la arteriola aferente.

La adición de furosemida causó una reducción significativa e intensa de un $82 \%$ en las resistencias aferentes de los animales control (Fig. 3A), y de un $65 \%$ en las de los tratados con CsA, que siguieron elevadas en torno a los valores basales. En los animales control, la reducción de las resistencias aferentes aumentó significativamente el FPR (Fig. 3B). En los animales tratados con CsA, también se observó un aumento del FPR, pero fue de menor magnitud, alcanzando niveles similares a los basales (Fig. 3B). El efecto de furosemida sobre el FPR fue significativamente modificado por CsA.

En los animales control, el aumento del FPR inducido por furosemida aumentó significativamente la presión intraglomerular en un 28\%, y el FG en

El FG estimado por el ClCr no mostró diferencias significativas en los controles versus los tratados con CsA (Fig. 2C). Consecuentemente, la fracción de filtración mostró ser algo más alta en los animales tratados, pero las diferencias no alcanzaron significación estadística $(22,6 \pm 3,4 \%$ vs $28,6 \pm 1,4 \%$, NS).

La reducción del FSR en ausencia de cambios significativos en la PA media sugiere un aumento en las resistencias intrarrenales. Éstas fueron significativamente más altas (más de dos veces) en los animales tratados con CsA respecto a los animales control (Fig. 2D). El principal componente de estas resistencias parece estar en las resistencias preglomerulares (arteriola aferente), que fueron casi tres veces más altas en los animales tratados con CsA (Fig. 2E). En cambio, las resistencias eferentes no presentaron diferencias significativas entre ambos grupos (Fig. 2F).

El aumento de tono de la arteria aferente forma parte de un reflejo intrarrenal conocido un $48 \%$ (Figs. 3C y 3D). Ambos efectos fueron significativos. Sin embargo, en los animales tratados con CsA, la furosemida apenas aumentó la presión intraglomerular un $2 \%$ y el FG no presentó tampoco variaciones significativas (Figs. 3C y 3D).

Estos resultados sugieren un aumento en la retroalimentación túbulo glomerular desde el asa de Henle en los animales tratados con CsA, apuntando a cambios en la función tubular como causa de las alteraciones hemodinámicas intrarrenales observadas.

\section{Parámetros de función tubular}

Los animales control sometidos a las condiciones experimentales indicadas, presentan una EF de $\mathrm{Na}^{+}$mayor del 1\%. En cambio, los tratados con CsA presentan a los 15 dias una EF de $\mathrm{Na}^{+}$ menor del 1\% ( $p=0,006)$ (Fig. 4A). El estudio de localización de esta mayor reabsorción tubular, mostró diferencias importantes en la reabsorción 


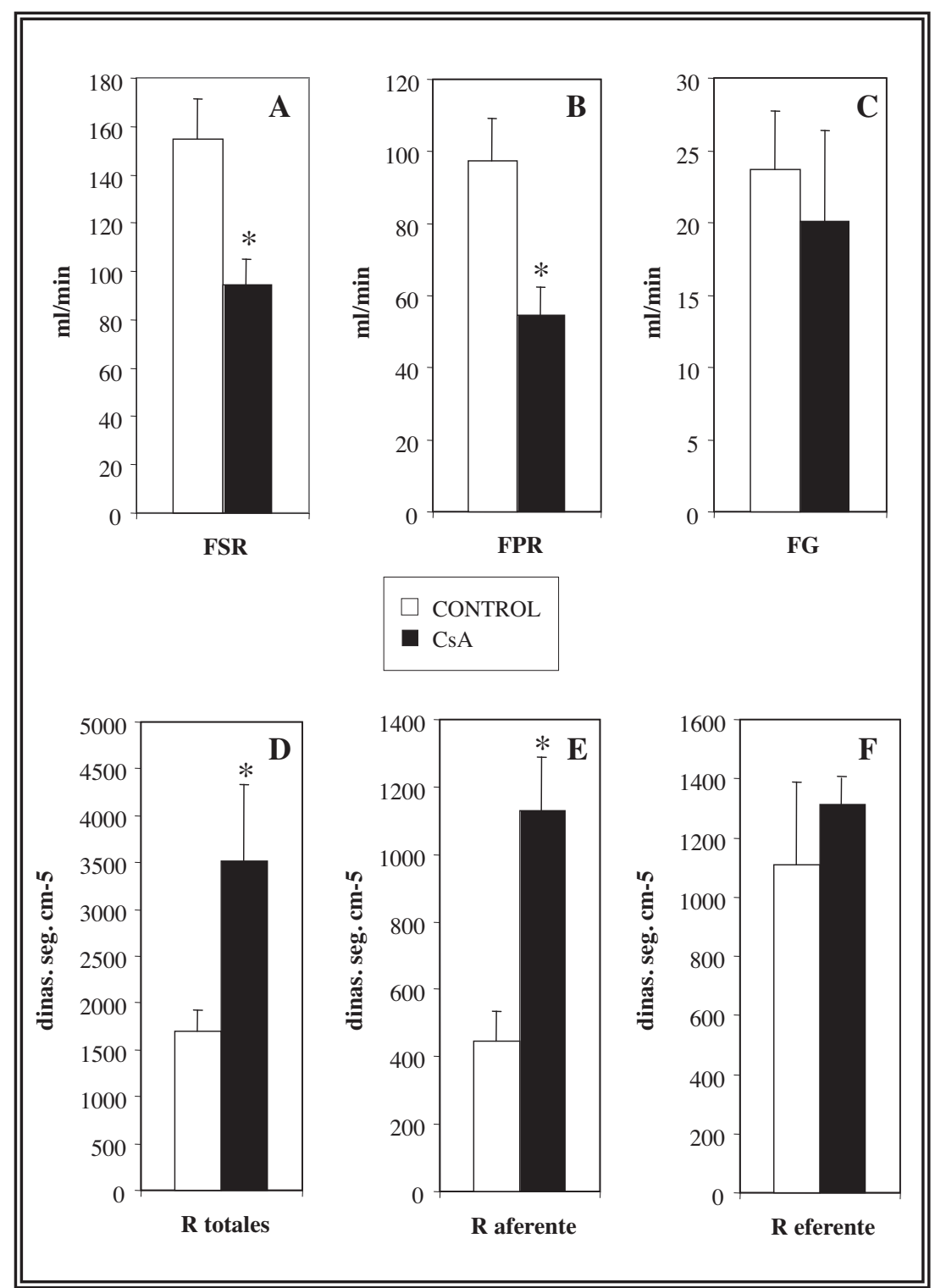

FIGURA 2. Efecto de la ciclosporina sobre la hemodinámica renal. Panel A: flujo sanguíneo renal medio. Panel B: flujo plasmático renal medio. Panel C: tasa de filtrado glomerular medio estimado por el aclaramiento de creatinina. Panel D: resistencias totales renales. Panel E: resistencias intrarrenales atribuibles a las arteriolas aferentes. Panel F: resistencias intrarrenales atribuibles a las arteriolas eferentes, en animales control $(n=7)$ y animales tratados durante 15 dias con ciclosporina $10 \mathrm{mg} / \mathrm{Kg}$.dia (n=6). * $p<0,05$, vs. control, " $t$ " de Student.

fraccional atribuible al túbulo distal. En los animales control, la $\mathrm{RFdCl}^{-}$era menor del $1 \%$, mientras que en los animales tratados con CsA subió por encima del 10\% ( $p=0,022)$ (Fig. 4B).

Como era de esperar, la furosemida inhibió la $\mathrm{RFdCl}^{-}$en ambas situaciones (Fig. 4B), aumentando significativamente la excreción fraccional de $\mathrm{Na}^{+}(\mathrm{p}<0,005)$ (Figura 4A).
La inhibición distal del trasporte de $\mathrm{Cl}^{-}\left(\mathrm{y} \mathrm{Na}^{+}\right)$por furosemida, aumenta la oferta de ambos a los sitios de intercambio del $\mathrm{Na}^{+}$por $\mathrm{K}^{+}$(túbulo colector cortical). Es de esperar que un aumento de flujo urinario a dicho nivel reduzca el gradiente observable de $\mathrm{K}^{+}$desde el capilar hasta la luz tubular (gradiente transtubular de $\mathrm{K}^{+}$). La furosemida redujo significativamente el GTTK en los animales control, pero no lo hizo en los animales que habian sido tratados con CsA (Fig. 4C).

La relación entre la reabsorción fraccional distal de $\mathrm{Cl}^{-}$y la excreción de $\mathrm{K}^{+}$muestra el funcionamiento de la nefrona distal donde la excreción de $\mathrm{K}^{+}$es inversamente proporcional a la reabsorción de $\mathrm{Cl}^{-}$. Puede observarse cómo en los animales control la $\mathrm{RFdCl}^{-}$nunca supera el 5\%. En cambio en los animales tratados con CsA, las excreciones de $\mathrm{K}^{+}$más bajas se corresponden con las $\mathrm{RFdCl}^{-}$más elevadas (Fig. 5).

\section{Consumo de oxígeno}

Las evidencias mostradas sugerian que los animales tratados con CsA tenían un aumento en la reabsorción nefronal de $\mathrm{Na}^{+}$, y que este aumento podría ser especialmente notable a nivel del asa de Henle. Esta variación en los sitios preferentes de reabsorción de $\mathrm{Na}^{+}$se exploró estudiando la estequiometría global renal aparente entre el consumo de $\mathrm{O}_{2}$ y el trasporte de $\mathrm{Na}^{+}$.

Al comparar ambos parámetros en los dos grupos sometidos a estudio, los valores de consumo de $\mathrm{O}_{2}$ y reabsorción de $\mathrm{Na}^{+}$se distribuyeron en torno a dos rectas de regresión cuyas pendientes fueron significativamente diferentes entre sí, consumiendo los animales tratados con CsA el doble de $\mathrm{O}_{2}$ que los animales control para reabsorber cantidades equivalentes de $\mathrm{Na}^{+}(\mathrm{p}<0,05)$ 


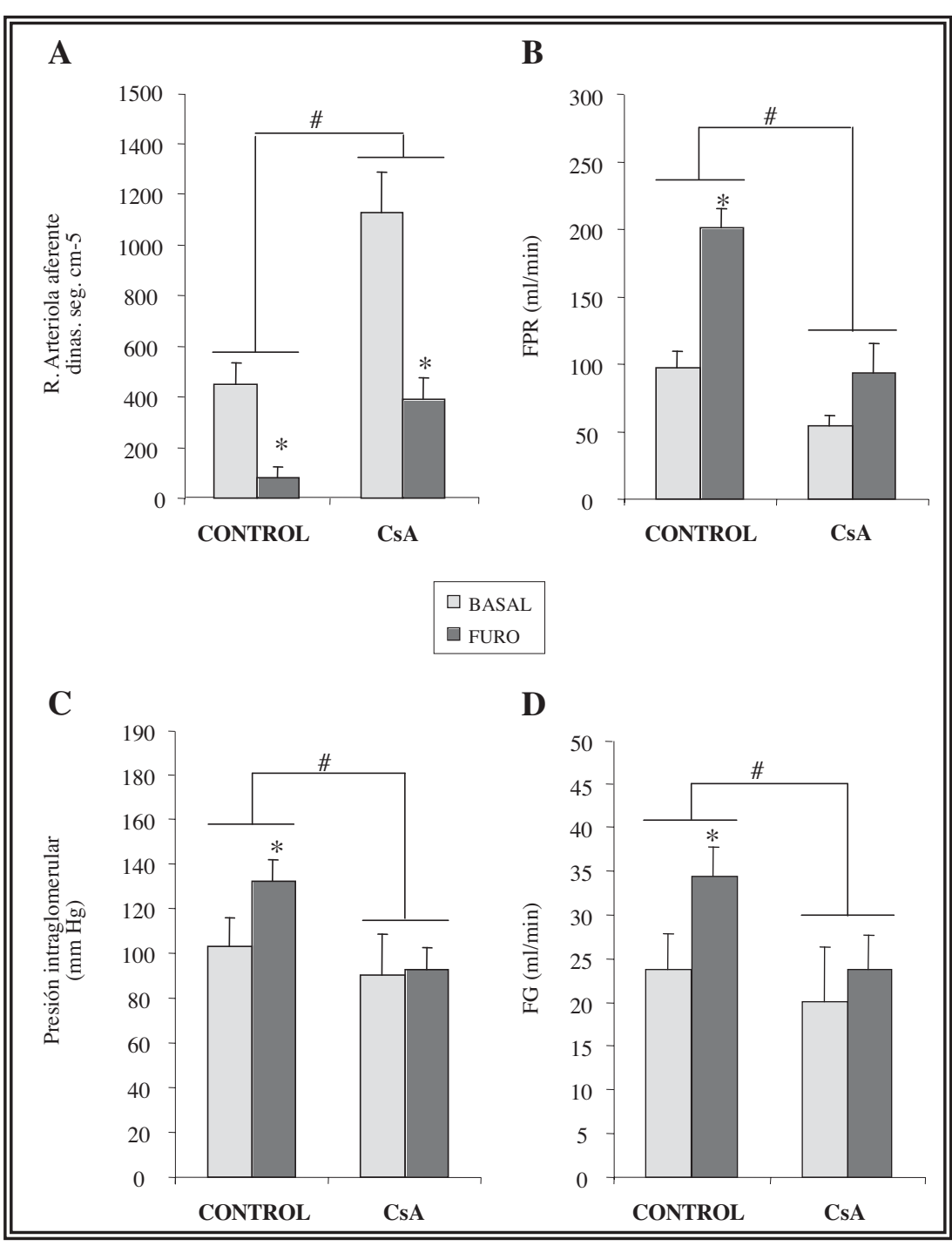

FIGURA 3. Efecto de la ciclosporina sobre la inhibición de la retroalimentación túbulo-glomerular por furosemida. Panel A: reducción de resistencias en la arteriola aferente inducidas por furosemida. Efecto del tratamiento con ciclosporina. ANOVA de dos factores para medidas repetidas, * variable "furosemida", $p=0,036$. \# variable "tratamiento con CsA", $p=0,012$; interacción "furosemida-tratamiento con CsA"; $p=0,303$. Panel B: aumento del flujo plasmático renal inducido por furosemida. Efecto del tratamiento con ciclosporina. ANOVA de tres factores para medidas repetidas. "efecto "furosemida", $p=0,001$; \# efecto "tratamiento con CsA", $p=0,024$. Panel C: aumento de la presión media intraglomerular secundaria a la reducción de resistencias aferentes y aumento del flujo plasmático renal inducidos por furosemida. Efecto del tratamiento con ciclosporina. ANOVA de dos factores para medidas repetidas: "efecto "furosemida", $p=0,023$; \# efecto "tratamiento con CsA", p=0,046; efecto de la interacción "furosemida-tratamiento CsA", $p=0,443$. Panel D: aumento del filtrado glomerular secundario al efecto de furosemida. Efectos del tratamiento con ciclosporina. ANOVA de dos factores para medidas repetidas. "efecto "furosemida", $p=0,027$; \# efecto "tratamiento con CsA", $p=0,231$; efecto de la interacción "furosemida-tratamiento con CsA", p=0,234. males tratados respecto a los controles (Fig. 6C).

La estequiometría media del consumo de $\mathrm{O}_{2} /$ transporte de $\mathrm{Na}^{+}$fue significativamente mayor en los animales tratados con $\mathrm{CsA}$, que en los controles (Fig. 6B).

\section{Actividad y localización de la} $\mathrm{Na}^{+}, \mathbf{K}^{+}$-ATPasa:

El estudio de actividad específica de la $\mathrm{Na}^{+}, \mathrm{K}^{+}$-ATPasa en microsomas procedentes de la corteza y de la médula renal de animales control o sometidos al tratamiento con CsA, mostraron una mayor actividad específica en la médula con respecto a la corteza. El tratamiento con CsA inhibió significativamente la actividad $\mathrm{Na}^{+}, \mathrm{K}^{+}$-ATPasa de la corteza, pero no de la médula renal (Fig. 7). La identificación de las tres subunidades estudiadas de la $\mathrm{Na}^{+}, \mathrm{K}^{+}-$ ATPasa, $\alpha 1, \beta 1$ y $\gamma$, mostraron resultados similares en los tres casos. A nivel del túbulo proximal pero no en el resto de los segmentos de la nefrona, la $\mathrm{Na}^{+}, \mathrm{K}^{+}$-ATPasa presenta una distribución intracelular diferente en los animales control y en los tratados con CsA. Mientras que en la situación control la distribución es la esperada por toda la vertiente basolateral, en los animales tratados la $\mathrm{Na}^{+}, \mathrm{K}^{+}$-ATPasa aparece distribuida por todo el citoplasma, sin un enriquecimiento basolateral claro. E1 borde basolateral aparece específicamente libre de $\mathrm{Na}^{+}, \mathrm{K}^{+}$-ATPasa. En la Figura 8 se presentan las imágenes para la tinción de la subunidad $\gamma$. Se obtuvieron datos similares para las subunidades $\alpha 1$ y $\beta 1$ (datos no mostrados).

\section{DISCUSIÓN}

(Fig. 6A). El mismo resultado se obtuvo para la relación entre el FSR y el consumo de $\mathrm{O}_{2}$, donde cada variación en $100 \mathrm{~mL} / \mathrm{min}$ del FSR provocó un mayor consumo de $\mathrm{O}_{2}$ por parte de los ani-
Nuestro modelo se basa en la administración de CsA 10 mg/Kg.día vía oral a cerdos miniatura, durante 15 dias, al cabo de los cuáles son evi- 


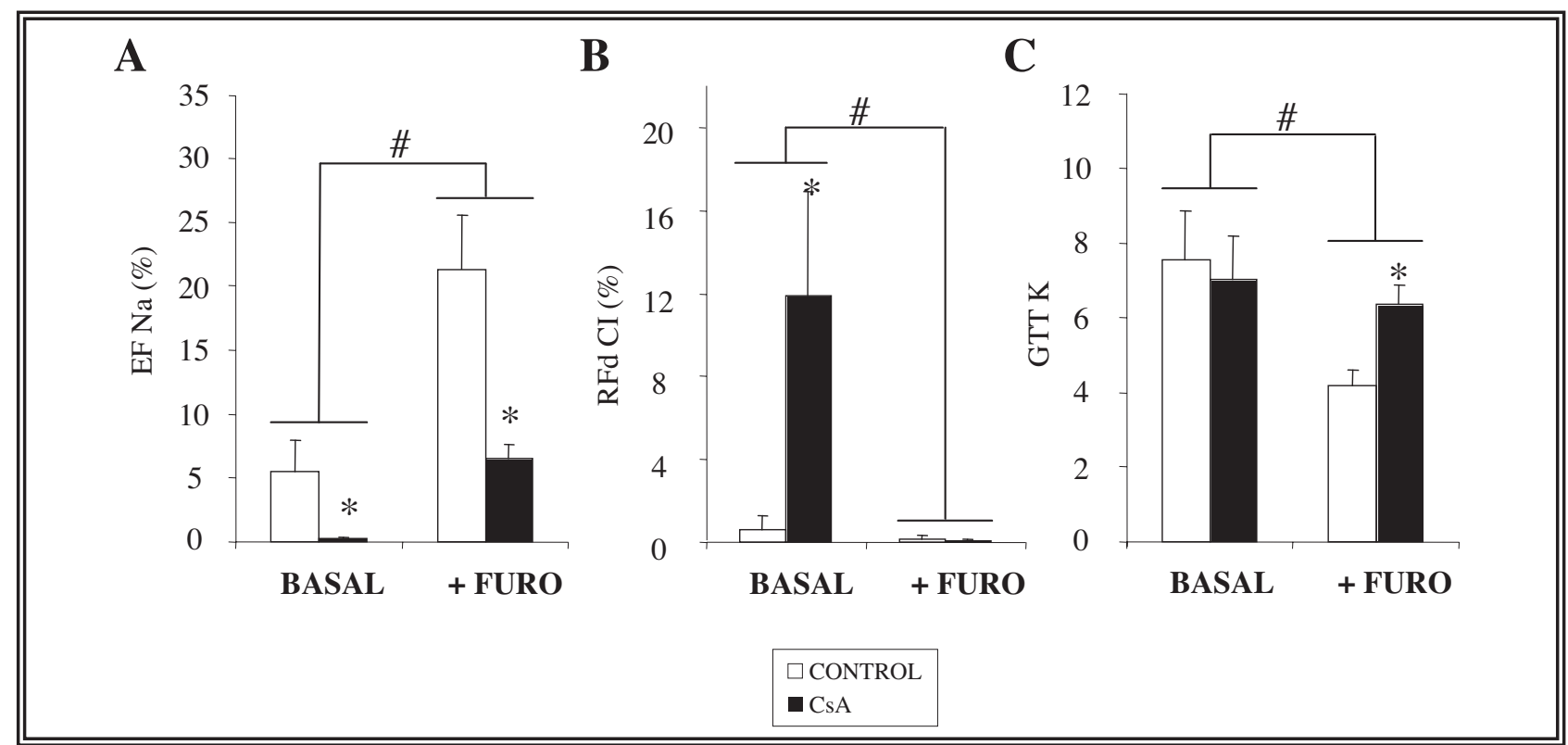

FIGURA 4. Efecto de la ciclosporina sobre la función tubular renal. Panel A: respuesta de la excreción fraccional a furosemida. Modificación por la ciclosporina. ANOVA de dos factores para medidas repetidas. * Efecto "furosemida", p<0,0005; \# efecto "tratamiento con CsA", $p=0,006$; efecto de la interacción "furosemida-tratamiento con CsA", $p=0,004$. Panel B: respuesta de la reabsorción fraccional de Cl-por el túbulo distal a la furosemida. Modificación por la ciclosporina. ANOVA de dos factores para medidas repetidas. * Efecto "furosemida", p=0,018; \# efecto "tratamiento con CsA", p=0,022; efecto de la interacción "furosemida-tratamiento con CsA", p=0,028. Panel C: respuesta del gradiente transtubular distal de $K+$ a la furosemida. Modificación por la ciclosporina. ANOVA de dos factores para medidas repetidas. * Efecto "furosemida", $p=0,045$; \# efecto "tratamiento con CsA", $p=0,458$; efecto de la interacción "furosemida-tratamiento con CsA", p=0,155.

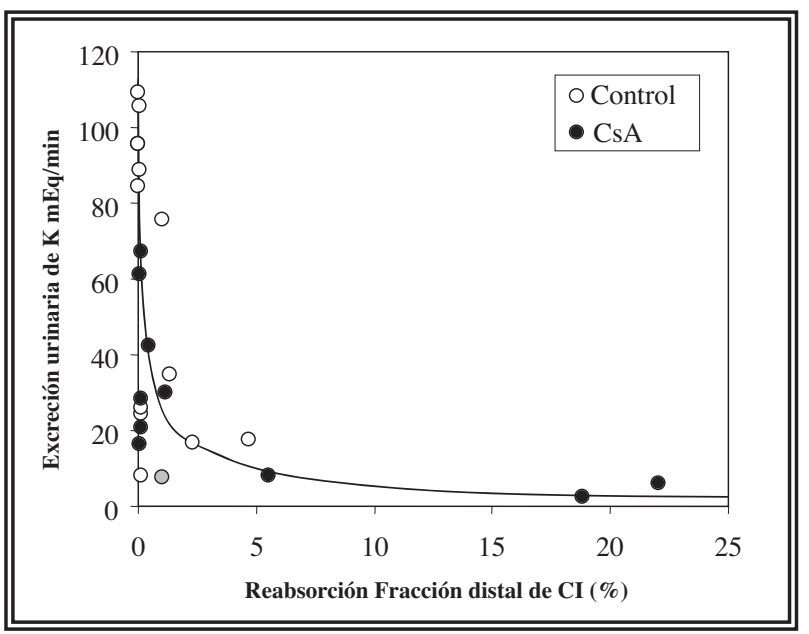

FIGURA 5. Funcionamiento del túbulo distal. Representación de las excreciones urinarias de $K+$ de cada animal frente a las reabsorciones fraccionales de $\mathrm{Cl}$ - por la nefrona distal. El comportamiento de los animales "Control" es diferente del de los tratados con CsA. La RFdCl- es siempre inferior al $5 \%$ y existe una relativa independencia entre la excreción urinaria de $K+$ y la RFdCl-. En los animales tratados con CsA, las RFdCl- más altas se corresponden con las potasiurias más bajas.

dentes cambios renales funcionales y estructurales, a pesar de que las adaptaciones hemodinámicas producidas impiden ver cambios significativos en el FG.
En presencia de CsA, la PA a nivel de la aorta no varió, pero aumentaron las resistencias aferentes de modo notable, produciendo una reducción en la presión de filtración. En publicaciones previas hemos mostrado que CsA, igual que FK506, es capaz de inhibir la sintesis de NO renal, tanto por la NOS constitutiva como por la inducible ${ }^{3}$. El tono de la arteriola aferente está controlado principalmente por el balance entre NO y endotelina, si bien otros agentes como catecolaminas y adenosina pueden también actuar a este nivel. Una inhibición de la síntesis de NO permite esperar un aumento de resistencias aferentes, que es precisamente lo que nuestros datos muestran. El efecto beneficioso de los anticálcicos sobre la toxicidad de CsA se puede explicar en parte porque la acción de los anticálcicos es fundamentalmente vasodilatadora sobre la arteriola aferente ${ }^{12}$.

En presencia de CsA, también aumenta el tono eferente. El aumento de resistencias eferentes es mucho menos significativo que el aumento de aferentes, pero parece suficiente como para que el FG no descienda de modo proporcional a la reducción en la presión glomerular. Consecuentemente, la fracción de filtración apenas 


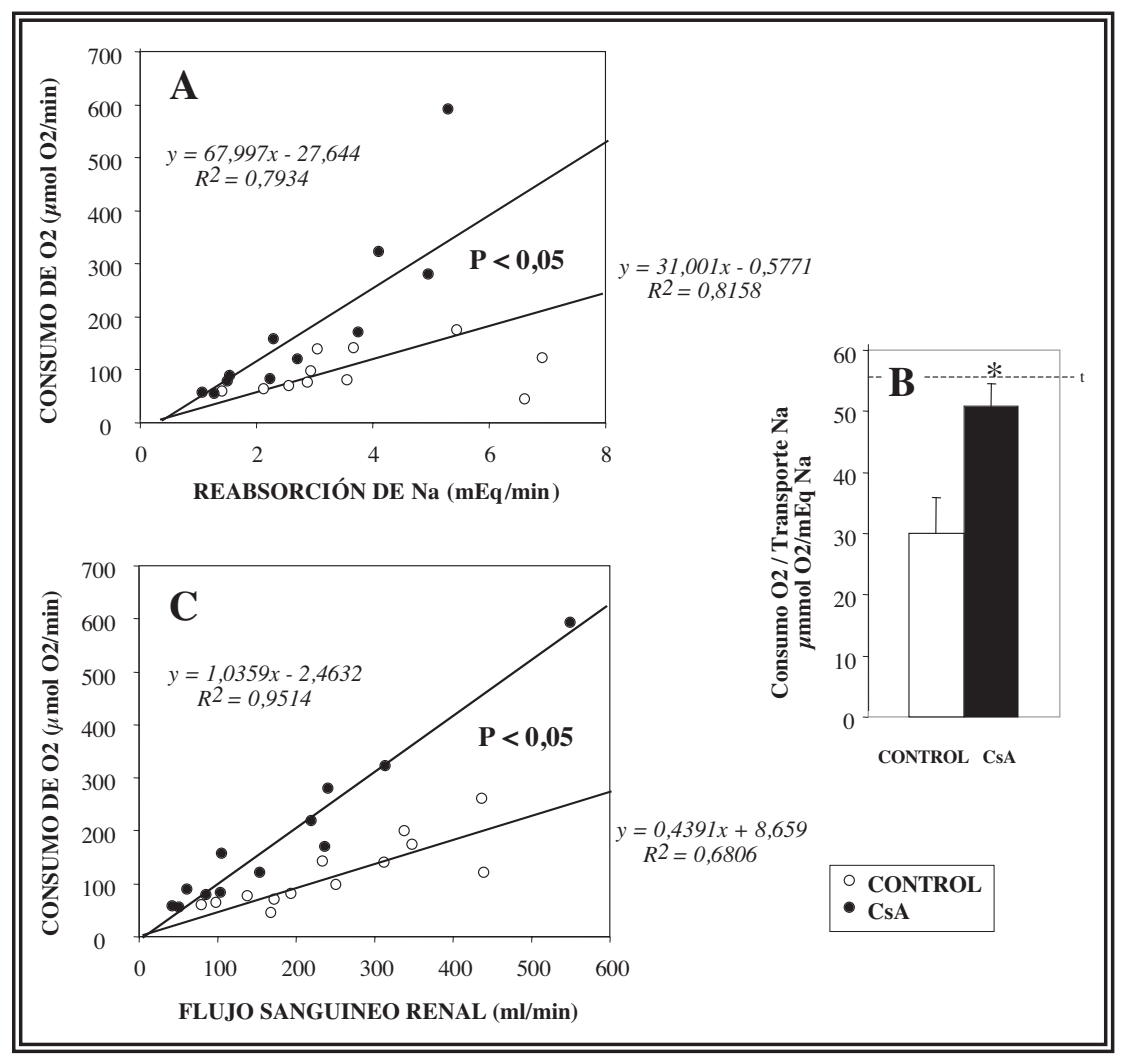

FIGURA 6. Panel A: relación entre el consumo real de 02 y el transporte de Na+. Los animales control se agrupan en torno a una linea de regresión cuya pendiente es significativamente diferente de la línea de regresión que agrupa ambos parámetros en los animales tratados con CsA. Panel B: estequiometría consumo de 02/transporte de $\mathrm{Na}+$ en los animales control y en los tratados con CsA. Los valores experimentales hallados coinciden con los coeficientes de las rectas de regresión determinadas en el panel A. Limite t: valor teórico máximo de un epitelio/órgano cuyo transporte de $\mathrm{Na}+$ es totalmente activo. Panel C: relación entre el consumo real de 02 y el flujo sanguíneo renal: los animales tratados con CsA presentan un comportamiento significativamente diferente de los controles. Para iguales flujos sanguíneos, el consumo de 02 de los primeros es significativamente más alto que el de los segundos.

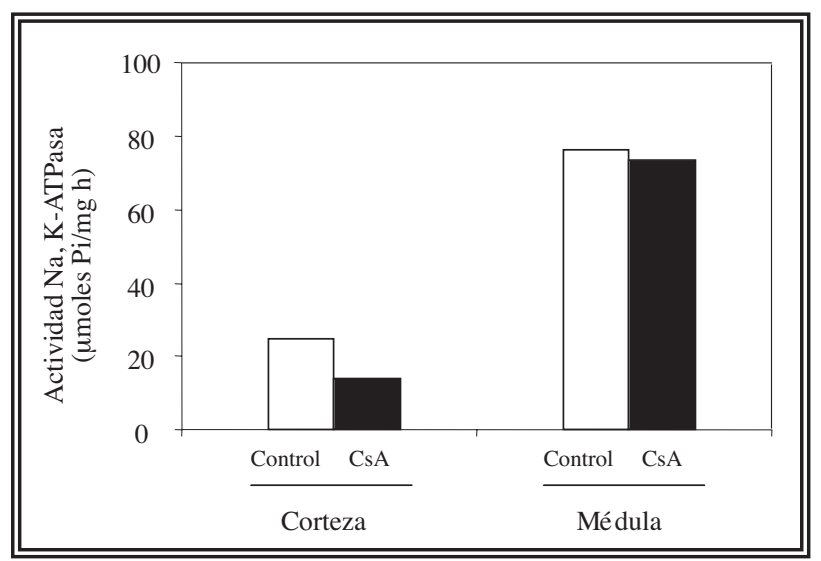

FIGURA 7. Actividad de la $\mathrm{Na}+, \mathrm{K}+-\mathrm{ATPasa}$ de microsomas aislados de la corteza o de la médula renal de animales control o animales tratados durante 15 dias con CsA $10 \mathrm{mg} / \mathrm{Kg} /$ dia. varía $(21,5 \% \pm 3,8$ vs. $27,4 \% \pm 1,6)$. Así pues, en el aumento global de resistencias intrarrenales observado con CsA, un potencial aumento de angiotensina II, o una inhibición preferente de la sintesis de prostaglandinas, no parecen jugar un papel prioritario.

El tono de la arteriola aferente forma parte del reflejo renal conocido como "feed-back" túbulo-glomerular o retroalimentación túbulo-glomerular (RTG) que se produce cuando en una nefrona aumenta el flujo y el trasporte a través del asa ascendente gruesa de Henle. Como consecuencia se produce vasoconstricción aferente en respuesta a un aumento de trasporte de $\mathrm{Na}^{+}, \mathrm{K}^{+}$y Cl-13.

Así pues, uno de los mecanismos potenciales por los que CsA puede estar causando un aumento en las resistencias aferentes es un aumento en la RTG. Para explorar esta posibilidad hemos utilizado furosemida. Furosemida inhibe el transportador apical $\mathrm{Na}^{+}: \mathrm{K}^{+}: 2 \mathrm{Cl}^{-}$ del asa ascendente de Henle, inhibiendo la RTG.

En los animales control, la adición de furosemida produjo en los 30 min siguientes la caída de resistencias aferentes, que se siguió de un aumento significativo del FPR, de la presión media intraglomerular $\mathrm{y}$, consecuentemente, del FG. Los animales tratados con CsA también redujeron sus resistencias aferentes con furosemida, pero sólo alcanzaron unos niveles de resistencias similares a las basales de los animales control. Consiguientemente, el FPR aumentó, pero los aumentos en presión media intraglomerular y FG no fueron significativos, alcanzando ambos parámetros valores similares a los basales de los animales no tratados. Así pues, la RTG era sólo parcialmente inhibida por furosemida.

Nuestros estudios previos in vitro han mostrado que CsA es capaz de inducir apoptosis sobre las células del túbulo proximal ${ }^{2}$, y los estudios 


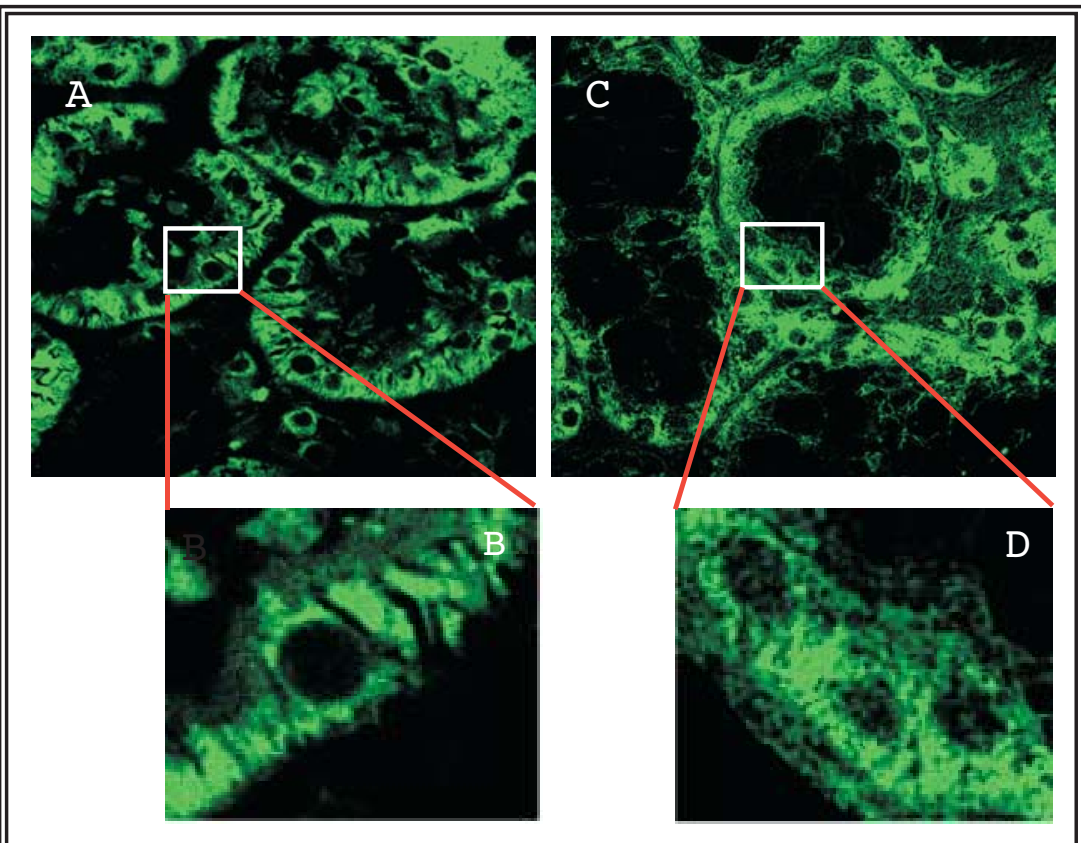

FIGURA 8: Inmunolocalización de la $\mathrm{Na+,K+-ATPasa}$ en las células del túbulo proximal. Riñones procedentes de animales control $(A, B)$, o tratados con CsA $10 \mathrm{mg} / \mathrm{Kg} /$ dia $\times 15$ dias $(C, D)$. Se ha utilizado una tinción con anticuerpos monoclonales especificos dirigidos contra la subunidad $\gamma$ de la bomba de sodio. Obsérvese en los detalles (B, control; $D, C s A)$ las diferencias en la distribución de la bomba de sodio. Mientras que en los animales control la distribución es basolateral, en los animales tratados con CsA, la bomba de Na+ aparece distribuida homogéneamente por todo el citosol sin refuerzo aparente a nivel basal, que aparece libre de tinción.

morfológicos también apuntan al túbulo proximal como diana fundamental de la toxicidad por $\mathrm{CsA}^{14}$. Incluso los efectos hemodinámicos de CsA implican la inhibición de la sintesis tubular de NO por $\mathrm{CsA}^{3}$. Sin embargo, no existen estudios acerca de las consecuencias que de esta disfunción proximal puede tener sobre el funcionamiento del resto de la nefrona.

En una aproximación simplista, una disfunción proximal podría acompañarse de una pérdida aumentada de $\mathrm{Na}^{+}$urinario. Sin embargo, la EF de $\mathrm{Na}^{+}$en los animales control, claramente superior al $1 \%$, cae significativamente a valores por debajo del $1 \%$ con CsA, remedando lo observado en clínica humana. Si el túbulo proximal está dañado, y sin embargo la EF de $\mathrm{Na}^{+}$está reducida, forzosamente otros segmentos nefronales están aumentando la reabsorción de $\mathrm{Na}^{+}$y $\mathrm{Cl}^{-}$.

Los animales tratados durante 15 dias con CsA presentan $\mathrm{RFdCl}^{-}$casi nueve veces superior a la de los controles. El parámetro estimado $\mathrm{RFdCl}^{-}$incluye mayoritariamente la reabsorción en el asa de Henle y túbulo distal, ya que a nivel del colector cortical, la permeabilidad al cloro es baja y juega un papel fundamental en la excreción urinaria de $\mathrm{K}^{+15}$. La inhibición del transportador $\mathrm{Na}^{+}: \mathrm{K}^{+}$: $2 \mathrm{Cl}^{-}$con furosemida reduce máximamente la $\mathrm{RFdCl}^{-}$, tanto en controles como en los animales tratados.

Uno de los hallazgos más significativos relativos a la nefrotoxicidad por CsA en clínica humana, relacionada con potenciales efectos tubulares distintos de los efectos descritos a nivel proximal, es la aparición precoz de hiperpotasemia, acompañada o no de acidosis, con bajas excreciones urinarias de $\mathrm{K}^{+}$. Cuando la hiperpotasemia acompaña a una reducción en el FG de pacientes trasplantados sometidos a tratamiento con CsA, suele considerarse como un signo altamente sugestivo de toxicidad por $\mathrm{CsA}^{16}$.

Después de 15 días de tratamiento con CsA, no observamos cambios en el $\mathrm{K}^{+}$plasmático, pero la excreción media de $\mathrm{K}^{+}$fue próxima a la mitad. Dado que casi todo el $\mathrm{K}^{+}$que aparece en orina procede de la secreción a nivel del segmento colector cortical de la nefrona, el GTTK permite valorar dicha función. No encontramos diferencias en el GTTK entre controles y CsA, pero cuando se inyecta furosemida el aumento de oferta distal de $\mathrm{Na}^{+}$y $\mathrm{Cl}^{-}$inducido por la inhibición del transporte en el asa ascendente debe aumentar la excreción urinaria de $\mathrm{K}^{+} \mathrm{y}$ el flujo urinario distal, reduciendo las diferencias de concentración entre el $\mathrm{K}^{+}$plasmático y el $\mathrm{K}^{+}$en la luz tubular (GTTK). Los animales controles redujeron adecuadamente su GTTK, pero no los tratados con CsA. Esta dificultad importante en la respuesta del túbulo colector cortical al intercambio de $\mathrm{Na}^{+}$por $\mathrm{K}^{+}$no puede ser atribuida a un hipoaldosteronismo primario ni secundario, ya que las determinaciones de aldosterona y renina mostraron que ambas hormonas están aumentadas en el grupo tratado con CsA (Tabla 1). 
Algunos autores han planteado la posibilidad de que CsA cause un aumento en la permeabilidad al cloro del túbulo colector cortical, produciendo una situación similar al Síndrome de Gordon ${ }^{17}$. La mayor permeabilidad al $\mathrm{Cl}^{-}$haría que el trasporte de $\mathrm{Na}^{+}$se siguiese de reabsorción de $\mathrm{Cl}^{-}$, no generando el gradiente de potencial negativo intraluminal necesario para la secreción de $\mathrm{K}^{+}{ }^{15}$. La furosemida desenmascararía este defecto. A favor de esta hipótesis van los datos mostrados en la Figura 5, donde al representar la excreción urinaria de $\mathrm{K}^{+}$en función de la $\mathrm{RFdCl}^{-}$ se puede ver cómo todos los valores se disponen en torno a una hipérbola, en la que al aumentar la $\mathrm{RFd} \mathrm{Cl}^{-}$, disminuye la excreción urinaria de $\mathrm{K}^{+}$. Todos los controles tienen $\mathrm{RFd} \mathrm{Cl}^{-}$inferiores al $5 \%$, pero no los animales tratados con CsA, en los que las excreciones urinarias de $\mathrm{K}^{+}$más bajas se corresponden con las mayores $\mathrm{RFd} \mathrm{Cl}^{-}$.

$\mathrm{Si}$ nuestra interpretación de los datos es correcta, una inhibición del trasporte de $\mathrm{Na}^{+}$a nivel del túbulo proximal con desplazamiento axial de una porción considerable de la carga filtrada y aumento de la reabsorción de $\mathrm{Na}^{+}$en la nefrona distal (asa de Henle, túbulo distal y colector distal) tendría repercusiones importantes en el consumo renal de oxígeno.

El trasporte de sodio en el túbulo proximal obedece a la reabsorción secundariamente activa de bicarbonato y otras especies (glucosa, aminoácidos, fosfato) acoplados al trasporte de $\mathrm{Na}^{+}$. Pero el grueso del trasporte obedece a fuerza de difusiones pasivas motivadas por el aumento progresivo de la concentración de cloro en la luz, que acababa siendo trasportado hacia el capilar por vías paracelulares, arrastrando consigo agua y electrolitos. Aproximadamente del 40 al 60\% del trasporte de $\mathrm{Na}^{+}$en el túbulo proximal es activo. A nivel del asa de Henle todo el trasporte de $\mathrm{Na}^{+}$ se basa en el funcionamiento de la bomba de sodio, y el porcentaje de trasporte activo es mucho mayor.

Asumiendo que una molécula de oxígeno produce a nivel mitocondrial seis moléculas de ATP, y que cada molécula de ATP utilizado por la bomba de sodio trasporta tres moléculas de $\mathrm{Na}^{+}$, la estequiometría teórica de un epitelio cuyo trasporte de $\mathrm{Na}^{+}$fuese totalmente dependiente de bombas de sodio sería de 18 moléculas de $\mathrm{Na}^{+}$ trasportadas por cada molécula de oxígeno. En otras palabras, el trasporte de $1 \mathrm{mmol} \mathrm{de} \mathrm{Na}^{+}$en dicho epitelio requeriría 56 mmoles de $\mathrm{O}_{2}$, si el epitelio sólo tuviese trasporte activo.

Al relacionar el trasporte renal de $\mathrm{Na}^{+}$con el consumo de $\mathrm{O}_{2}$ por el riñón, observamos que los datos procedentes de los animales control se distribuian en torno a una recta con una pendiente de 31 mmoles de $\mathrm{O}_{2}$ consumido por $1 \mathrm{~mol} \mathrm{de} \mathrm{Na}^{+}$ trasportado. Este dato coincidía con el valor medio de la estequiometría calculada en cada animal 30,0 \pm 5,9 mmoles de $\mathrm{O}_{2} / \mathrm{mmol}$ de $\mathrm{Na}^{+}$. Sin embargo, todos los animales tratados con CsA presentaban consumos renales de oxígeno mayores (a pesar de la reducción en FSR), y sus datos se agrupaban en torno a una recta diferente, con una pendiente de 67 mmoles de $\mathrm{O}_{2}$ consumido por $1 \mathrm{~mol} \mathrm{de} \mathrm{Na}^{+}$trasportado, semejante a la estequiometría media determinada en cada animal: 50,9 \pm 3,6 mmoles de $\mathrm{O}_{2} / \mathrm{mmol} \mathrm{de} \mathrm{Na}^{+}$, ambos notablemente próximos al valor teórico de 56 atribuible a epitelios con trasporte de $\mathrm{Na}^{+}$ mayoritariamente activo como el asa ascendente de Henle.

La confirmación de que este fenómeno se está produciendo en la realidad la obtuvimos al estudiar la bomba de sodio en los túbulos proximales y en las asas de Henle procedentes de animales control y tratados con CsA. Mientras que en el túbulo proximal CsA (y FK506) producían inhibición de la actividad de la bomba de sodio, la actividad de la misma en el asa de Henle permanecería inalterada. En el túbulo proximal de los animales tratados con $\mathrm{CsA}$ la $\mathrm{Na}, \mathrm{K}$-ATPasa no presentaba la distribución basolateral característica, apareciendo distribuida por todo el citoplasma. Hallazgos similares han sido comunicados con la lesión del túbulo proximal durante la isquemia reperfusión ${ }^{18}$.

Por último, no hemos encontrado diferencias en el manejo del agua que sugieran trastornos a nivel del túbulo colector medular o papilar.

En conclusión, CsA determina un aumento de resistencias de la arteriola aferente que reduce el flujo sanguíneo y plasmático renal, y la presión intraglomerular. La retroalimentación túbulo glomerular está aumentada, aparentemente debido a un desplazamiento axial en la carga de sodio procedente del túbulo proximal, primariamente 
dañado por CsA. El túbulo colector cortical presenta una secreción defectuosa de $\mathrm{K}^{+}$, que se pone de manifiesto tras sobrecargas del segmento. La respuesta del filtrado glomerular, las resistencias intrarrenales y el gradiente transtubular de $\mathrm{K}^{+}$con furosemida están alterados en los animales tratados con CsA incluso en presencia de aclaramientos de creatinina no reducidos de modo significativo.

\section{Agradecimientos}

Este trabajo ha sido posible gracias a la financiación parcial de una beca de la Fundación Mutua Madrileña, del Fondo de Investigación Sanitaria (FIS 05/2259), y del Consorcio del Fracaso Renal Agudo (SBIO-0 283-2006, Comunidad de Madrid).

Nuestro agradecimiento a la colaboración técnica de M.A. Díaz, P. Tavares y J. García-Bordás.

\section{REFERENCIAS}

1. Flanagan WM, Corthésy B, Bram RJ, Crabtree GR. Nuclear association of a T-cell transcription factor blocked by FK506 and cyclosporin A. Nature. 1991;352(6338):803-807.

2. Perez M, Castilla M, Torres AM, Lazaro JA, Sarmiento E, Tejedor A. Inhibition of brush border dipeptidase with cilastatin reduces toxic accumulation of cyclosporin A in kidney proximal tubule epithelial cells. Nephrol Dial Transplant. 2004;19(10):2445-2455.

3. Hortelano S, Castilla M, Torres AM, Tejedor A, Bosca L. Potentiation by nitric oxide of cyclosporin A and FK506induced apoptosis in renal proximal tubule cells. J Am Soc Nephrol. 2000;11(12):2315-2323.

4. Sachs DH, Leight G, Cone J, Schwarz S, Stuart L, Rosenberg S. Transplantation in miniature swine. I. Fixation of the major histocompatibility complex. Transplantation. 1976;22(6):559-567.

5. Gomez DM. Evaluation of renal resistances, with special reference to changes in essential hypertension. J Clin Invest. 1951;30(10):1143-1155.

6. Navar LG. Regulation of renal hemodynamics. Advan Physiol Educ. 1998;275:221-235.

7. Kelman GR. Digital computer subroutine for the conversion of oxygen tension into saturation. J Appl Physiol. 1966;21 (4):1375-1376
8. Martínez Maldonado M, Schwartz A. Renal ATPase as a receptor for drugs acting on the kidney. En Martinez Maldonado M, director. Methods in Pharmacology. Nueva York: Plenum Publishing Co; 1976. P: 199-225.

9. Wetzel RK, Sweadner KJ. Immunocytochemical localization of Na-K-ATPase alpha- and gamma-subunits in rat kidney. Am J Physiol Renal Physiol. 2001;281(3):F531-545.

10. Gonzalez-Martinez LM, Avila J, Marti E, Lecuona E, Martin-Vasallo P. Expression of the beta-subunit isoforms of the Na,K-ATPase in rat embryo tissues, inner ear and choroid plexus. Biol Cell. 1994;81(3):215-222.

11. Mercer RW, Biemesderfer D, Bliss DP Jr, Collins JH, Forbush B 3rd. Molecular cloning and immunological characterization of the gamma polypeptide, a small protein associated with the Na,K-ATPase. J Cell Biol. 1993;121(3): 579-586.

12. Rodicio JL. Calcium antagonists and renal protection from cyclosporine nephrotoxicity: long-term trial in renal transplantation patients. J Cardiovasc Pharmacol. 2000;35(3 Suppl 1):S7-11.

13. Komlosi P, Fintha A, Bell PD. Current mechanisms of macula densa cell signalling. Acta Physiol Scand. 2004;181 (4):463-469.

14. Myers BD. Cyclosporine nephrotoxicity. Kidney Int. 1986; 30(6):964-974

15. Tejedor A. Fisiología Ácido-Base. En Ayus C, Caramelo C, Tejedor A, directores. Agua, Electrolitos y Equilibrio ÁcidoBase. Madrid: Editorial Médica Panamericana; 2007, p: 142-159.

16. Caliskan Y, Kalayoglu-Besisik S, Sargin D, Ecder T. Cyclosporine-associated hyperkalemia: report of four allogeneic blood stem-cell transplant cases.Transplantation. 2003;75(7):1069-1072.

17. Kamel KS, Ethier JH, Quaggin S, Levin A, Albert S, Carlisle EJ, Halperin ML. Studies to determine the basis for hyperkalemia in recipients of a renal transplant who are treated with cyclosporine. J Am Soc Nephrol. 1992;2(8):1279-1284.

18. Molitoris BA, Geerdes A, McIntosh JR. Dissociation and redistribution of $\mathrm{Na}+, \mathrm{K}(+)$-ATPase from its surface membrane actin cytoskeletal complex during cellular ATP depletion. J Clin Invest. 1991;88(2):462-469.

Correspondencia autor: Dr. A. Tejedor Jorge

Departamento de Nefrología. Laboratorio de Fisiopatología Renal Hospital General Universitario Gregorio Marañón

Dr. Esquerdo 46 - 28007, Madrid. Tel.: 914265145

E-mail autor: atejedor@nefro.hggm.es

Información artículo: Original 